\title{
Metal Detection by Multi-Component TEM Method
}

\author{
Chow-Son Chen ${ }^{1, *}$, Wei-Hsuan Chiu ${ }^{1}$, and Ching-Ren Lin ${ }^{1,2}$ \\ ${ }^{1}$ Institute of Geophysics, National Central University, Chung-Li, Taiwan, ROC \\ ${ }^{2}$ Institute of Earth Sciences, Academia Sinica, Taipei, Taiwan, ROC
}

Received 18 December 2007, accepted 23 May 2008

\begin{abstract}
During exploration using electromagnetic methods, secondary electromagnetic (EM) fields due to induced currents from conductors, together with the primary EM field, are recorded with a suitable receiver at various points in space. In general, the secondary EM field at the receiver, which contains all the information regarding underground conductors, may be several orders of magnitude smaller than the primary field. Under these conditions the separation of the measured total EM field into its primary and secondary parts is difficult. For the purpose of measuring the secondary fields in the absence of the primary field, time-domain electromagnetic measurements, often referred to as transient electromagnetic (TEM) techniques are employed.

Comparative numerical modeling using a single component (measuring the vertical component of the field, Hz) TEM receiver and a three-component (measuring $\mathrm{Hx}, \mathrm{Hy}$, and $\mathrm{Hz}$ ) TEM receiver was undertaken. A forward modeling approach was used to compute the voltage response of half-space containing one or more conductive bodies excited by a bi-polar square waveform. Although this method utilizes conductor scattering, it is particularly useful as a practical use for unexploded ordnance (UXO) detection. Unlike single component data, results from the three-component data are unambiguous as to the location and orientation of conductors. Measurements with the addition of horizontal- component data for secondary magnetic fields lead a better indication of target location, and target size determination, orientation, and characteristics, especially for the targets in the horizontal plan. A three-component TEM field experiment at a well-documented well site (NCU campus) was consistent with the effects predicted by our theoretical modeling. As a result, the three-component TEM survey is an essential element for high-resolution EM engineering survey.
\end{abstract}

Key words: Three-component TEM, Metal detection, Well field of NCU

Citation: Chen, C. S., W. H. Chiu, and C. R. Lin, 2009: Metal detection by multi-component TEM method. Terr. Atmos. Ocean. Sci., 20, 445-454, doi: 10.3319/TAO.2008.05.23.02(T)

\section{INTRODUCTION}

During exploration by traditional frequency-domain electromagnetic (FEM) methods, the ground is energized by passing an alternating current (ac) through an ungrounded loop typically situated at the earth's surface. The primary field of the loop will induce eddy currents in all conductors present in the earth. The secondary electromagnetic (EM) fields due to these induced currents, together with the primary EM field, are recorded with a suitable receiver at various points in space. In general, the secondary EM field at the receiver, which contains all the information regarding the underground conductors, may be several orders of magnitude smaller than the primary field. Under these conditions

\footnotetext{
* Corresponding author

E-mail:chusen@earth.ncu.edu.tw
}

the separation of the measured total EM field into its primary and secondary parts is difficult. This fact led to the idea of using time-domain electromagnetic measurements, often referred to as transient EM (TEM) techniques (Nabighian 1991). In TEM measurements a strong direct current $(\mathrm{dc})$ is usually passed through an ungrounded loop. At time $\mathrm{t}=0$ this current is abruptly interrupted. The secondary fields due to the induced eddy currents in the ground can now be measured with a suitable receiver in the absence of the primary field. For poor conductors, the initial voltages are large but the field decays rapidly. For good conductors the initial voltages are smaller but the field decays slower. This thus provides a simple criterion for recognizing and differentiating the effects of various conductors. 
In the detection of unexploded ordnance (UXO), nearsurface TEM surveys have traditionally used one or more $\mathrm{Hz}$ loops (measuring the vertical component of the field). However, in this paper, we offer a numerical model of TEM three-component $(\mathrm{Hz}, \mathrm{Hx}$, and Hy) responses for vertical and horizontal subterranean conductors. Based on this numerical modeling, we devise a three-component TEM field experiment at a well-documented wells site on the NCU campus. We find three-component TEM anomalies, which correlate with known wells, and which are consistent with the effects predicted by our theoretical modeling. We conclude that the three-component TEM survey is an essential tool for high-resolution EM measurements.

\section{THEORETICAL BACKGROUND AND MODELING}

In order to understand the TEM responses with a rectangular transmitter loop excited by a bi-polar square waveform with exponential rise-time and ramp turnoff, a forward modeling approach was used.

\subsection{Modeling Code}

LEROI is a well-known program designed to operate with a variety of field survey configurations and on single or multiple TEM readings using a forward approach. It is sponsored by a group of companies under the Australian Minerals' Industry Research Association (AMIRA). The program computes the voltage $(\mathrm{db} / \mathrm{dt})$ response of up to two horizontally layered half-spaces. The deeper or basement layer may contain one or more conductive plate-style bodies (Conductive Plate). The theoretical responses derived from these targets are inductively coupled.

LEROI is based upon frequency domain, integral equation thin sheet theory (Raiche and Sugeng 1989). This approach computes scattering currents from separate current gatherings and vortex potentials. The time domain response is computed by first calculating the frequency domain response from $0.1 \mathrm{~Hz}$ to $100 \mathrm{kHz}$ at an interval of six frequencies per decade. A cubic spline interpolation of the imaginary component of the magnetic field is used to represent the frequency domain response over the entire spectrum. The time domain magnetic fields are computed using digital filter coefficients that have been derived from the fast Hankel transform algorithm of Johansen and Sorensen (1979). A spline is fit to the time domain magnetic fields and the $\mathrm{db} / \mathrm{dt}$ computed using the spline coefficients. This is then convolved with the source function to give the field voltages (by area $* d b / d t)$.

In its theoretical implementation, LEROI decomposes a plate conductor into a series of sub-plates when modeling. These sub-plates are used to more accurately simulate the physical EM secondary fields created in an actual conductor.
By simulating small sub-plate cells the EM response computed can be more accurately computed but at the expense of computational time. A default cell size of 50 meters is used but for large plates, this may be increased. There is a compromise between EM response accuracy and computation time. The code is compute intensive and can be slow to model soundings in complex situations. Memory requirements for the program are high; it will not operate in less than 16 megabytes of available memory, but the program operates more efficiently when 32 megabytes of RAM is available. It will take about 2 minutes, Intel Pentium processor $1400 \mathrm{MHz}$, for modeling a plate in the conductive host (Figs. 1 and 3), with a cell size of 1 meter and turn off time (tot) of transmitting current of $0.140 \mathrm{~ms}$ (the tot record of our experiment).

\subsection{D Modeling}

Figure 1 shows the calculated profile plots from a plate (conductance $3000 \mathrm{~S}$ ) in the conductive host with a resistivity of $100 \mathrm{ohm}-\mathrm{m}$. Figure $1 \mathrm{~b}$ is the survey map showing the location of the plate and survey lines. Figure 1c shows the calculated TEM transient voltage (in $\mu \mathrm{v})$ along line $6(\mathrm{y}=6 \mathrm{~m})$ of $\mathrm{Hz}, \mathrm{Hx}$, and Hy components, top down, respectively. The conductive features are clearly anomalous with strong negative values relative to the background for the $\mathrm{Hz}$ profile; the calculated Hx anomalies are dipolar with a negative/crossover/positive anomaly as the target is approached and crossed; the calculated $\mathrm{Hy}$ anomalies are similar to $\mathrm{Hz}$ but the polarity of the anomaly is reversed. In each case, the cross-over point is centered over the target. Figure $1 \mathrm{~d}$ shows the calculated TEM transient voltage along line $10(\mathrm{y}=10 \mathrm{~m})$. The dominant difference is of no Hy anomaly since the y component is parallel to the plate, which is assumed to be of zero thickness. Figure 1e shows the calculated TEM transient voltage along line $14(\mathrm{y}=14 \mathrm{~m})$. The profile is quite similar to profile 6 , but the Hy component has opposite polarity.

We organized all the profiles and plot the results in planar view in Fig. 2 (e.g., picking delay time $2.087 \mathrm{~ms}$ ). Figure 2a shows the vertical component, while Fig. 2b shows the horizontal component (the composition of the $\mathrm{x}$ and $\mathrm{y}$ component). When compared to the plot between vertical and horizontal components, the horizontal component reveals more clearly the possible target conductor. The refinement of conductor images by adding the horizontal component is especially helpful for target identifications common at engineering sites.

For the case of the target conductor being horizontal, the calculated profile plots and planar plots are created as in Figs. 3 and 4, respectively. After examining and comparing these plots, it is evident that the horizontal component is better for horizontal target identification than the vertical component. This is due to the horizontal components being able to display the anomaly generated by the horizontally conductive target (Figs. 3c, d, and e). 
(a)

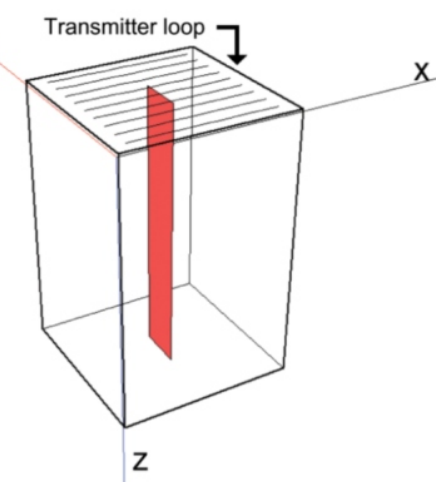

(b)

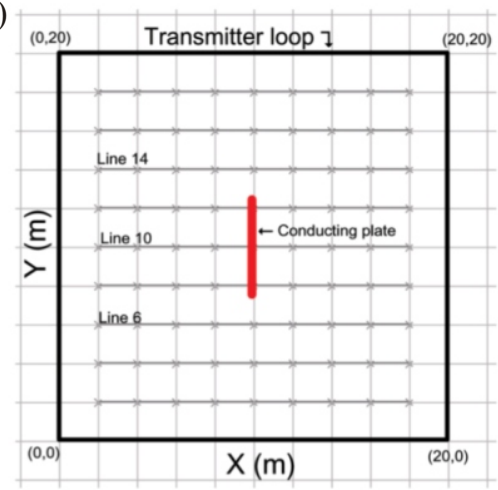

(c)

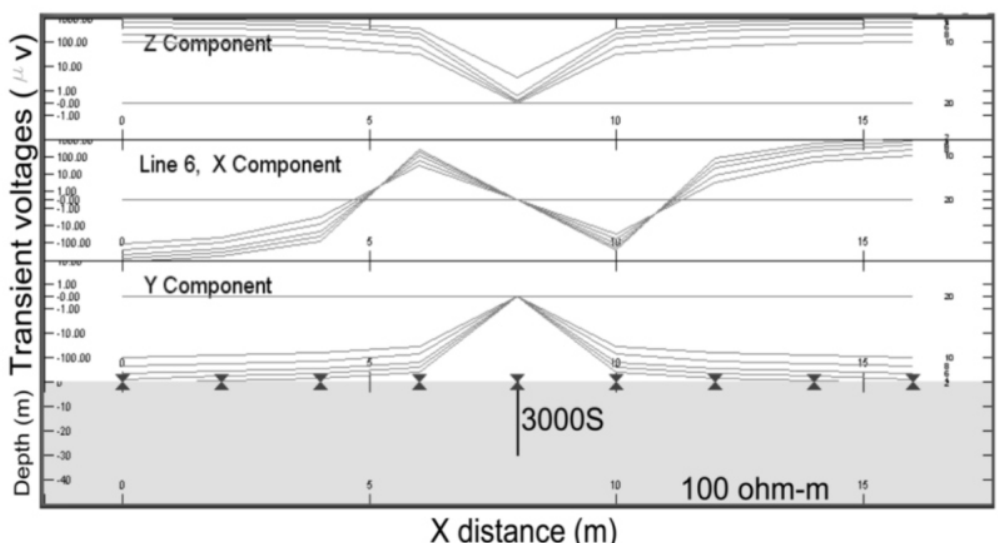

(d)

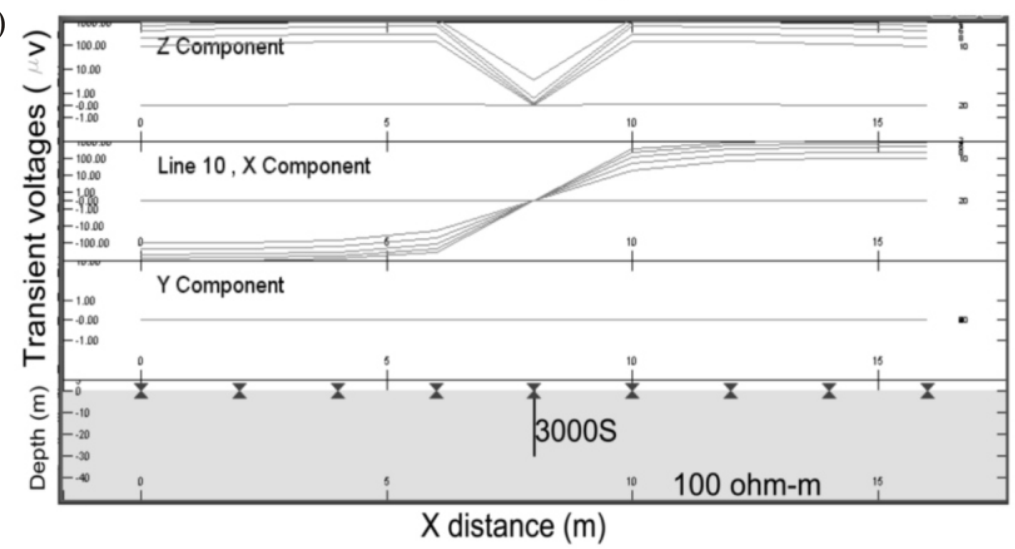

(e)

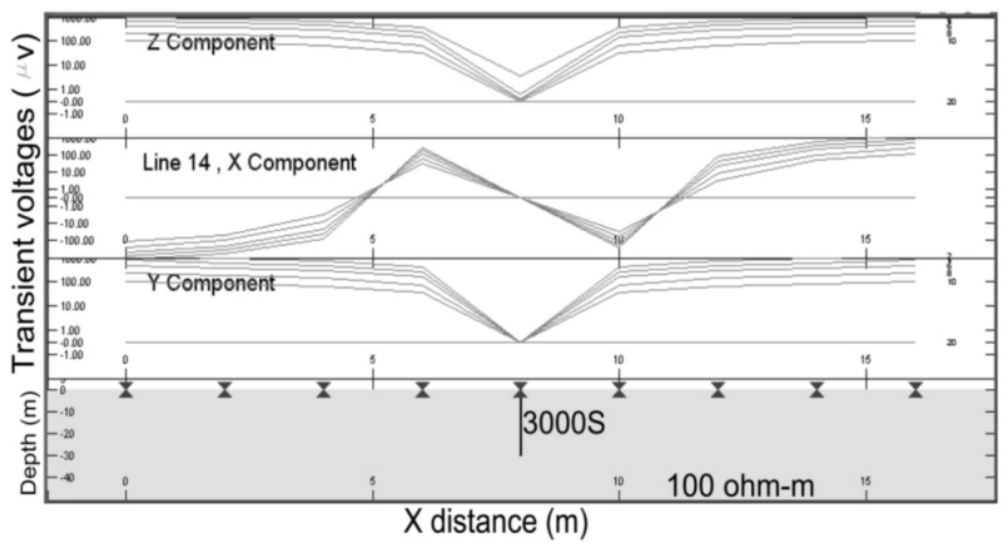

Fig. 1. Computed three-component transient voltages for the TEM profiles of a vertical plate (conductance $3000 \mathrm{~S})$ in a conductive host (100 ohm-m). (a) The perspective displays of the three-dimensional model. Transmitter loop position and survey lines are displayed on the top surface at ground level. Target plate suggests depth and three-dimensionality. (b) The survey map showing the location of the model plate, transmitter loop (bold square) and survey lines. (c) The calculated TEM transient voltage (in $\mu \mathrm{V}$ ) along line 6 of $\mathrm{Hz}, \mathrm{Hx}$, Hy component, and the earth model, top down respectively. (d) The calculated TEM transient voltage along line 10. (e) The calculated TEM transient voltage along line 14. 
(a)

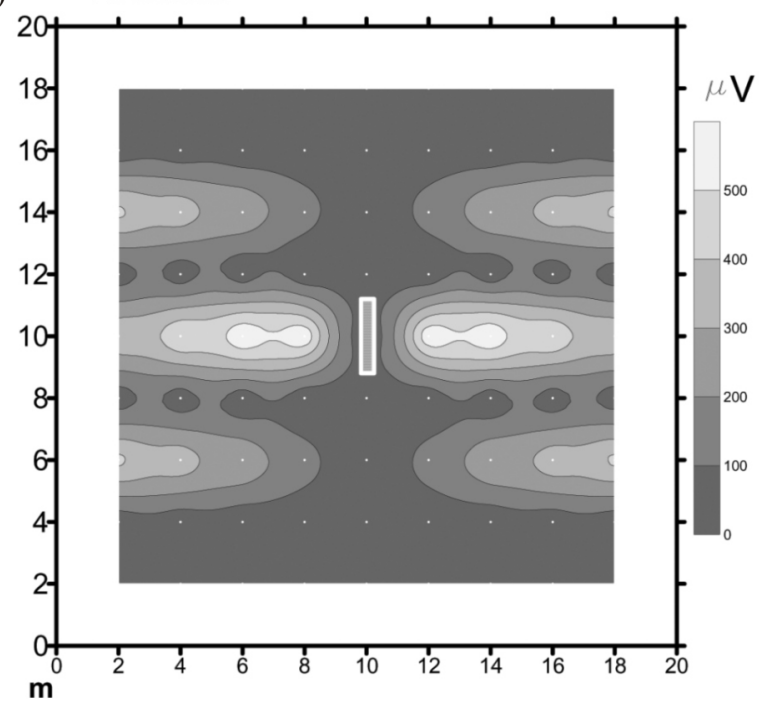

(b)

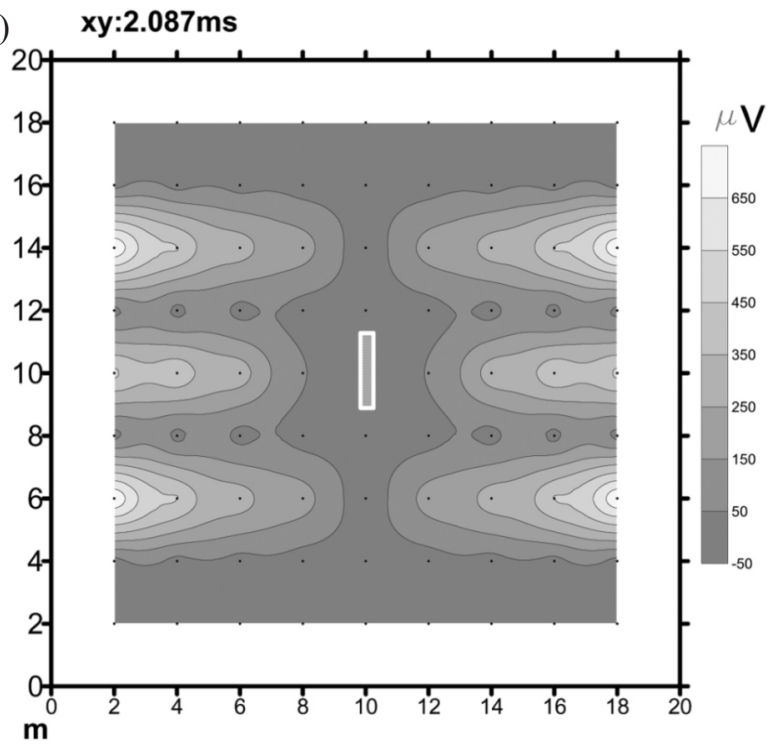

Fig. 2. Planar view of the TEM transient voltage (in $\mu \mathrm{V}$ ) at time $2.087 \mathrm{~ms}$ of Fig. 1. (a) Vertical component. (b) Horizontal component. The conductive target is indicated by a red box.

\section{THREE-COMPONENT TEM FIELD EXPERIMENTS}

A comparative survey using a three-component (measuring $\mathrm{Hx}, \mathrm{Hy}$, and $\mathrm{Hz}$ ) TEM receiver was undertaken at six wells in the Groundwater Research Well Field on NCU campus (Chen and Chang 2002), four of which have stainless steel casings and the other two plastic (Fig. 5a). The campus is located on the Taoyuan Tableland. Deposits consist of an upper sequence of a red-brown lateritic soil over rounded sandstone clasts (up to boulder size) in an uncemented matrix of sand, silt, and clay, and a lower sequence of interbedded, well-consolidated sand, silt, and clay. The lower clay layer is composed of laterally continuous clay from $34 \mathrm{~m}$ to the well-depths of $35 \mathrm{~m}$.

\subsection{Field Experiments}

For our three-component TEM survey, two sets of 50\% duty cycle, time domain, square wave electric current (each 25 amps) in parallel are transmitted into ungrounded two transmitter square loops of wire measuring $20 \mathrm{~m}$ on each side. In this survey, the magnetic fields are detected with a three-orthogonal ungrounded Roving Vector Receiver, each dipole moment equaling $10000 \mathrm{~m}^{2}$. The measurements are made 18 to 20 times ("windows"), from about 0.5 to $30 \mathrm{~ms}$, after the transmitter is turned off in order to measure the decay response of the background earth as well as secondary fields from conductors after the background earth response has decayed to near-zero values. SIROTEM TEM (Buselli and O'Neill 1977) is used for this purpose. An area measuring 20 meters by 20 meters around the central six wells in the
Groundwater Research Well Field is surveyed in detail to establish the extent of the subsurface metal, as well as to detect any other nearby anomalies.

\subsection{Data Analysis and Interpretation}

For suggested data analysis and interpretation of the TEM survey, we use contours of voltage for a selected number of channels of data: at least one early delay time giving shallow behavior, one late delay time giving deeper behavior, and one intermediate delay time for delineating or checking purposes. The anomalies are then rated. The choice of the optimum delay time at which measurements of the TEM response are made is based on the response of a given target being at its maximum for conductivity and radius. However, in the presence of geological noise, it is not the target alone that determines the optimum delay time at which TEM measurements are taken. In such cases, the optimum delay time is derived by given consideration to the behavior of the signal to noise ratio as a function of time, and determining when these functions reach a maximum. For our experimental case, within the time window $0.5-30 \mathrm{~ms}$, we chose early delay time of $\sim 1.687 \mathrm{~ms}$ and a late delay time of $\sim 5.087 \mathrm{~ms}$

Figure $5 \mathrm{~b}$ shows the $\mathrm{Hz}$ survey results in planar view at 1.687 and $5.087 \mathrm{~ms}$ after the transmitter is turned off. From the shallow image $(1.687 \mathrm{~ms})$, the subsurface metal casings at the well site are clearly anomalous as negative transient voltages. This closely correlates to the well site. Using modeling (Fig. 2), we are confident that the negative anomalies were due to the metal casings. With an increased depth image $(5.087 \mathrm{~ms})$, six anomalies associated with the well cas- 
(a)

$y$

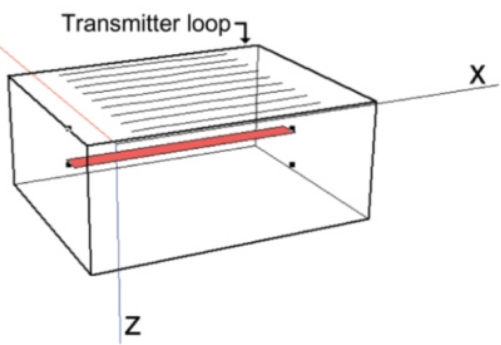

(b)

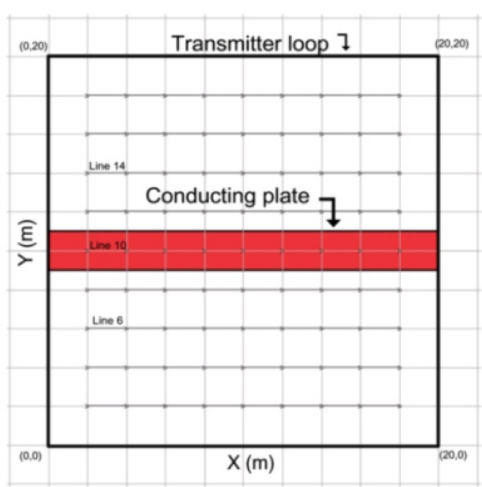

(c)

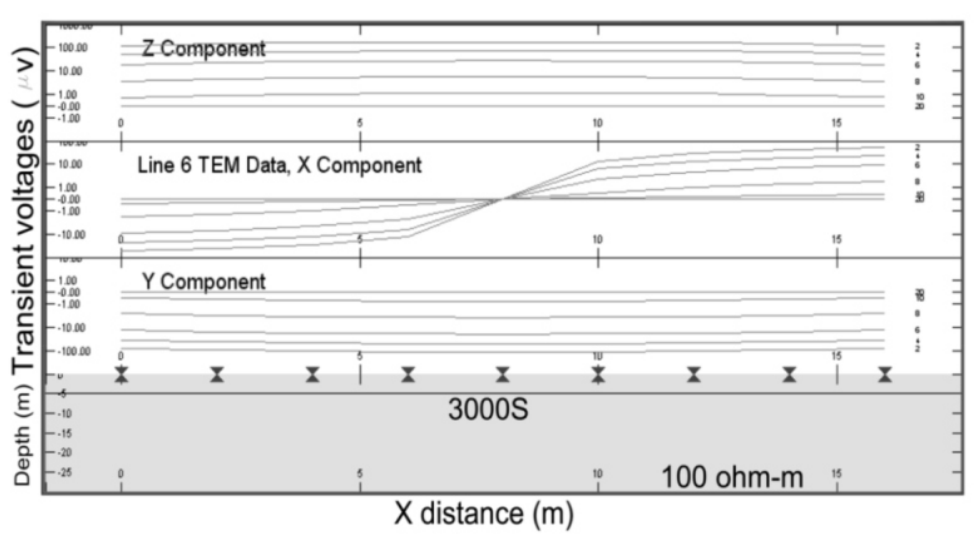

(d)

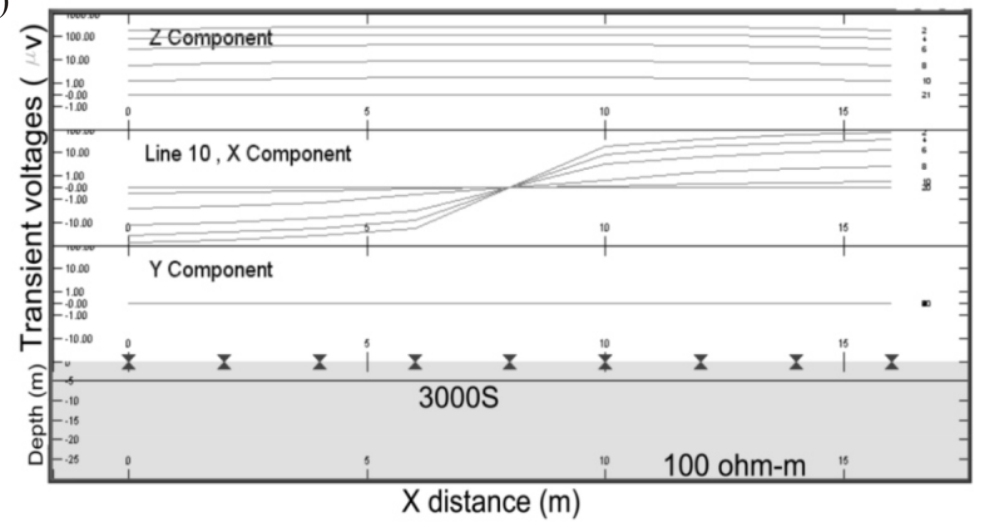

(e)

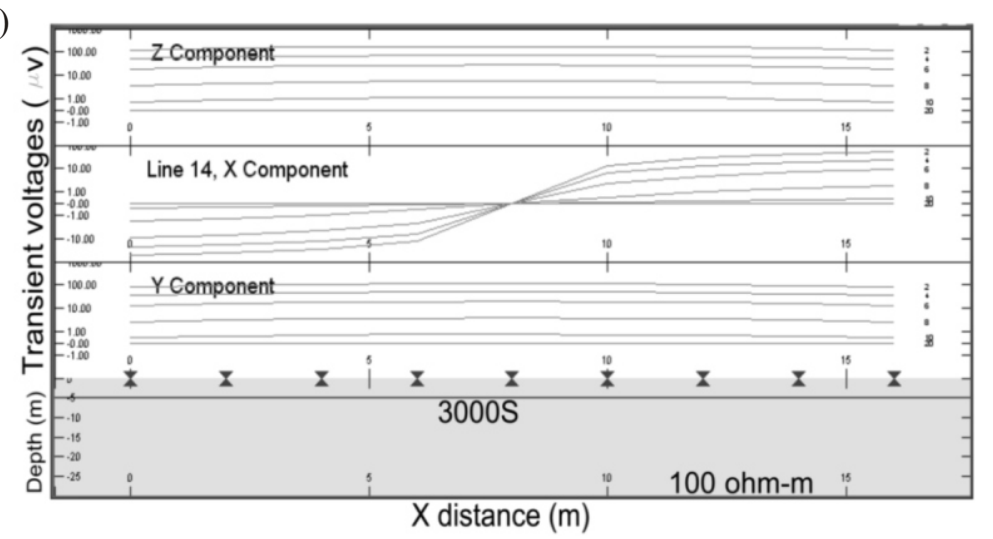

Fig. 3. Computed three-component transient voltages (in $\mu \mathrm{V}$ ) for the TEM profiles of a horizontal plate (conductance $3000 \mathrm{~S}$ ) in a conductive host (100 ohm-m). (a) The perspective displays of the model. Transmitter loop position and survey lines are displayed on the top surface at ground level. Target plate (in red color) suggests depth and three-dimensionality. (b) The survey map showing the location of the model plate (in red color), transmitter loop (bold square) and survey lines. (c) The calculated TEM transient voltage (in $\mu \mathrm{V}$ ) along line 6 of Hz, Hx, Hy components, and the earth model, top down respectively. (d) The calculated TEM transient voltage along line 10. (e) The calculated TEM transient voltage along line 14. 
(a)

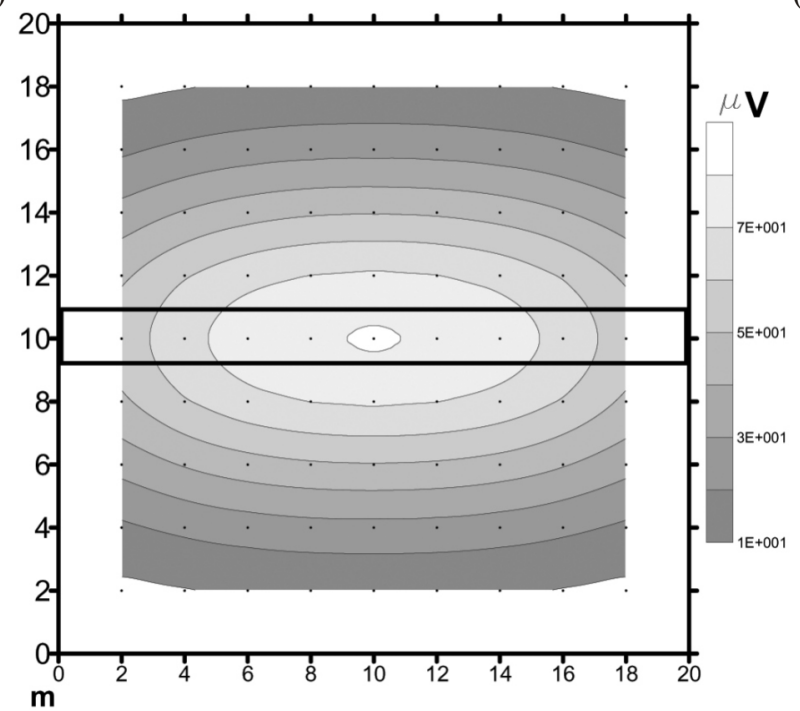

(b)

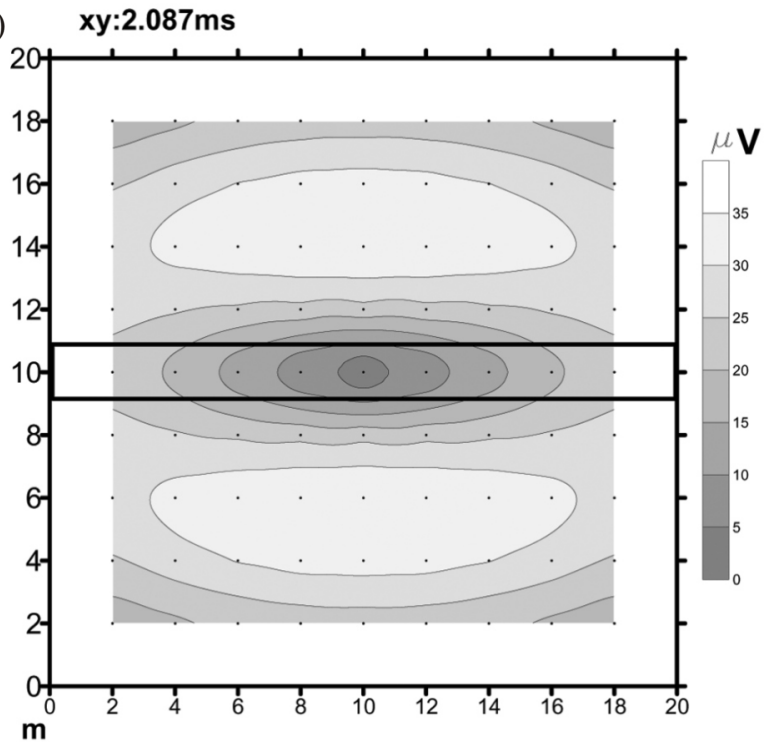

Fig. 4. Planar view of the TEM transient voltage (in $\mu \mathrm{V}$ ) at time $2.087 \mathrm{~ms}$ of Fig. 3. (a) Vertical component. (b) Horizontal component. The conductive target is indicated by a box.

ings are clearly seen. The response of well 3 is weaker leading us to suspect this well is one of those lacking a metal casing.

Other than metal identification beneath the earth, the characteristics of the surrounding earth could also be estimated from using TEM anomalies. Examining the two different depth slices of Fig. 5b, very conductive materials (negative voltages) surrounded the well casing at shallow depths $(1.687 \mathrm{~ms})$ while less or none conductive materials are evidenced at depth $(5.087 \mathrm{~ms})$. As the experimental targets were drilled using the cable tool percussion method, heavy drilling mud would have been used and we know that bentonite layers were deployed, to prevent cross flow between wells (Chen and Chang 2002). Both heavy drilling mud and bentonite layers are extremely conductive materials; therefore, the images of the metal casings are mixed with the very conductive materials of well construction. The extent of the depth of the very conductive materials can be estimated using the concept of skin depth in the time domain (Lee 1977). For a planar wave and a homogeneous medium, the maximum electrical field penetrates at depth $\mathrm{z}(\mathrm{m})$ :

$\mathrm{z}=(2 \mathrm{t} \rho / \mu)^{1 / 2}$

where $\mathrm{t}=$ time (s), $\rho=$ resistivity $(\mathrm{ohm}-\mathrm{m})$ and $\mu=$ magnetic permeability $(\mathrm{H} / \mathrm{m})$. Suppose the late time of $5.087 \mathrm{~ms}$ responds to a casing depth of $35 \mathrm{~m}$ according to Eq. (1), and the early time of $1.687 \mathrm{~ms}$ response depth is about $20 \mathrm{~m}$, which is the extent of the very conductive materials.

Figure 5c shows TEM response of the wells in the horizontal component, calculated from $\mathrm{Hx}$ and Hy data. Only metal-casing wells 1,2 , and 4 are closely correlated with
TEM anomalies at the well sites from shallow to deep; the plastic-casing wells 5 and 6 have weak TEM response signals. This demonstrates the importance and superiority of the horizontal TEM component in vertical target detection. In that, it is capable of illuminating the orientations and characters of a metal target. However, the horizontal signal is weaker than the vertical, consequently, we transmitted a higher current of $50 \mathrm{~A}$ in this experiment [the behavior was predicted by our theoretical modelings (Figs. 1 and 3)].

After extending the survey to the surrounding area ( $\sim 50 \mathrm{~m}$ to the west), similar anomalies were encountered in the horizontal component data but not in the $\mathrm{Hz}$ data. Figure 6a gives the location, and Fig. $6 \mathrm{~b}$ presents an east-west trending anomalous zone for early time crossing through the grid at $y$ $=16 \mathrm{~m}$, moving from west to east along the survey line. This linear anomaly is the result of a buried power line that enters the survey grid from the west, connects to an electrical access cover $(x=12 \mathrm{~m}, \mathrm{y}=16 \mathrm{~m})$, and exists the grid to the east journeying toward another light pole. Because the horizontal power line is very shallow, about $1 \mathrm{~m}$ below the surface, the anomaly was dismissed as time increased to $2.087 \mathrm{~ms}$. A strong anomaly at coordinate $\mathrm{x}=12 \mathrm{~m}, \mathrm{y}=16 \mathrm{~m}$ is an electrical access cover on the surface. It is worth noting that there is a weak TEM response for vertical component measurement (Fig. 6c). In fact, if only the vertical component were measured in this survey, as in the traditional exploration approach, then the risk of failure would be great.

\section{DISCUSSIONS}

For general TEM surveys, employing a horizontal transmitter loop is common. It is apparent that $\mathrm{Hz}$ data, which dis- 
(a)

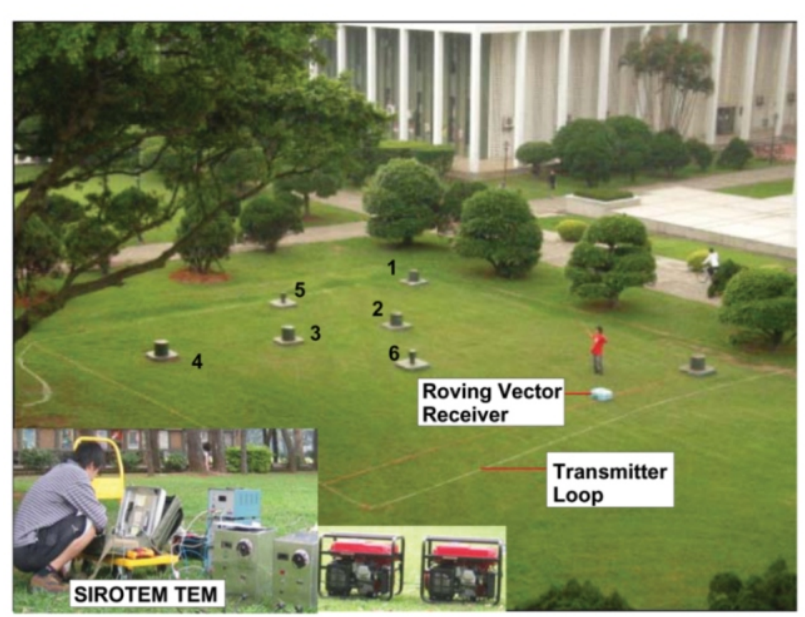

Well locations

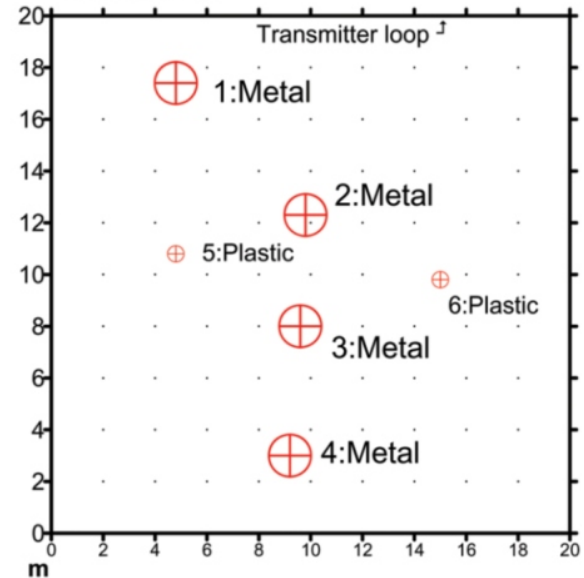

(b)

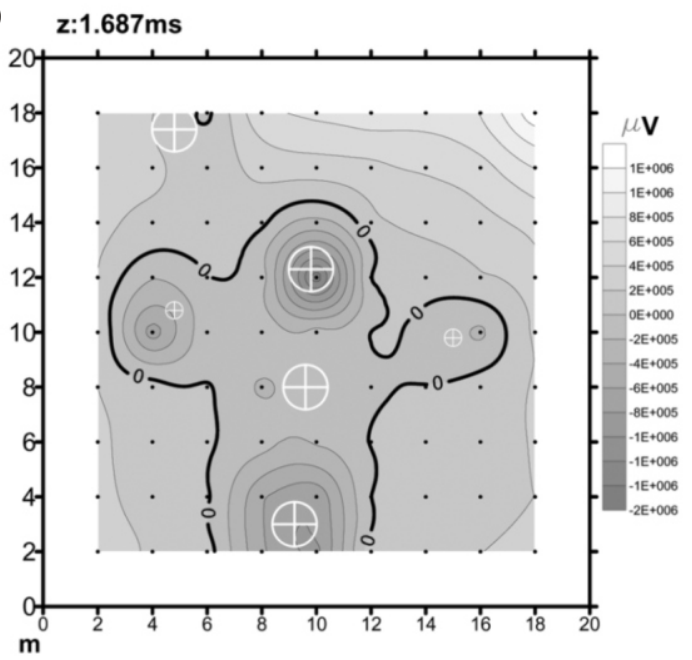

(c)

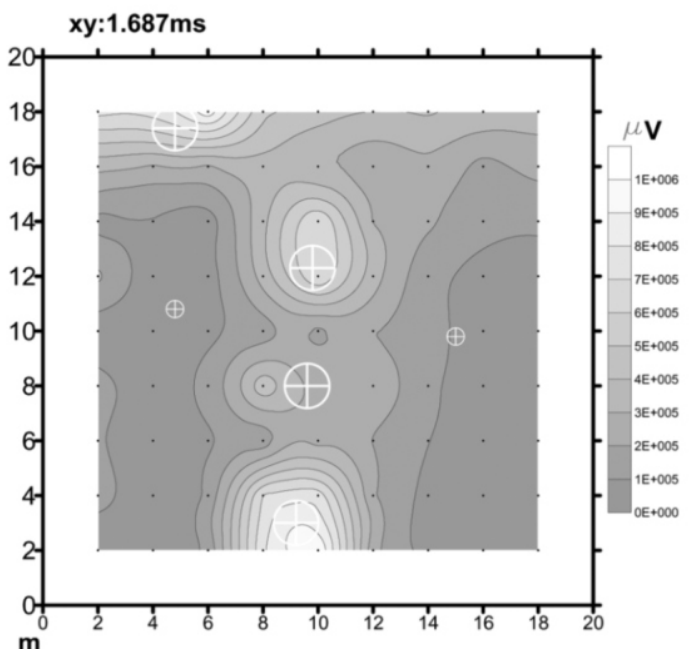

$\mathrm{z}: 5.087 \mathrm{~ms}$

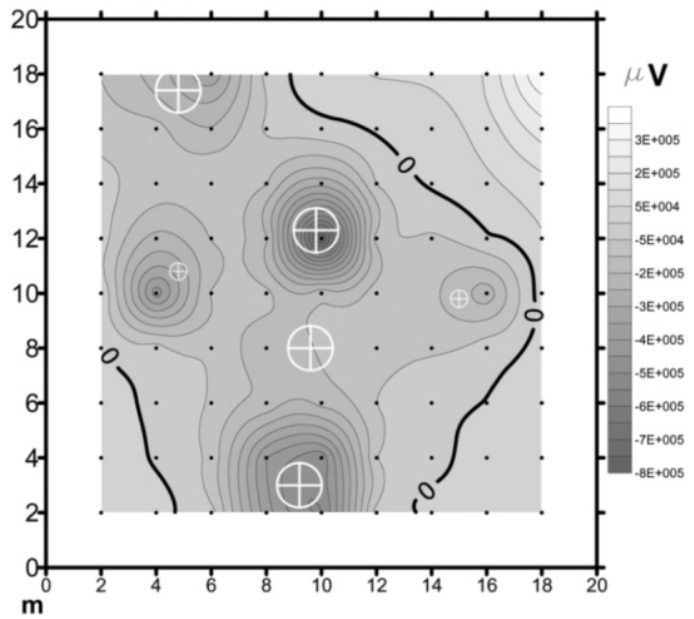

$x y: 5.087 \mathrm{~ms}$

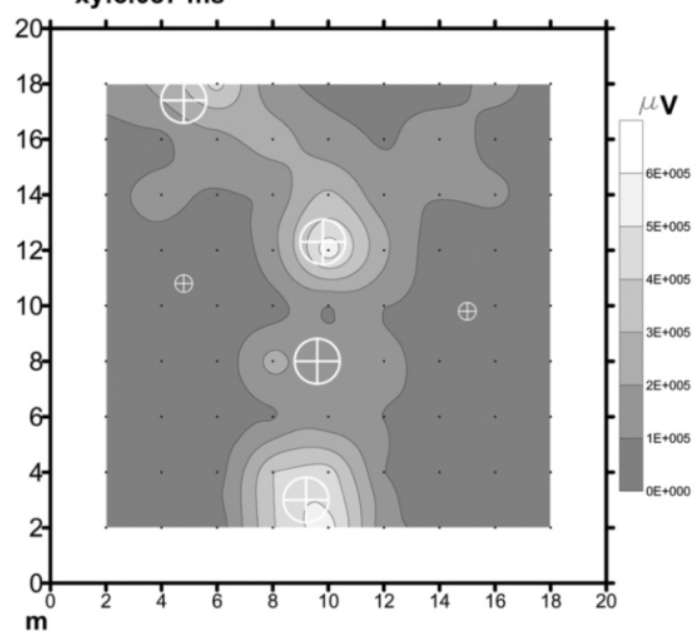

Fig. 5. Measured three-component transient voltages (in $\mu \mathrm{V}$ ) for the TEM map around the wells site on NCU campus. (a) Three-component TEM experiment at the Groundwater Research Well Field on NCU campus (Upper). The survey map showing the location of the TEM soundings (black dots) and the location of wells: the bigger cross for metal casing and the smaller one for plastic casing (Lower). (b) Vertical component TEM survey results in planar view around the well field at delay times 1.687 and 5.087 ms. Evidence of a strong anomaly near well locations. (c) Horizontal component TEM survey results in planar view for the same area at delay times 1.687 and $5.087 \mathrm{~ms}$. Compared with the vertical component, the horizontal component TEM anomalies are more responsive to the casing characteristics, metal and plastic. 
(a) Power line location

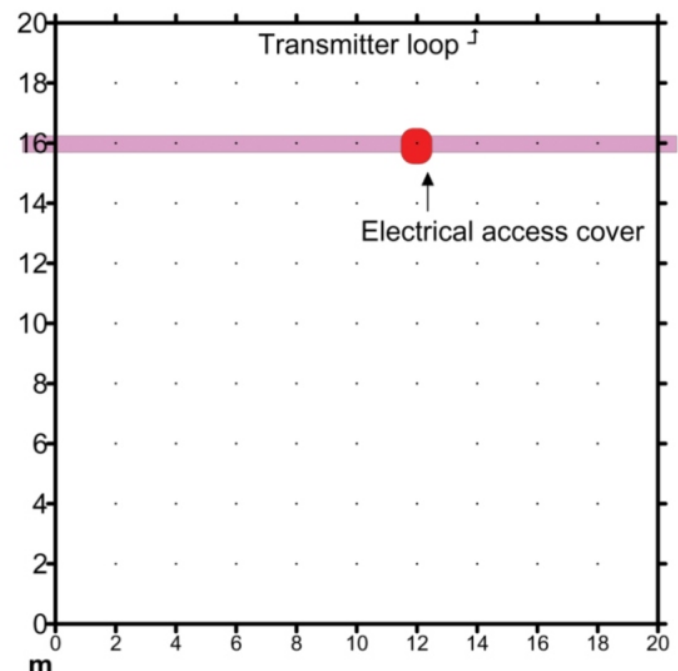

(b)

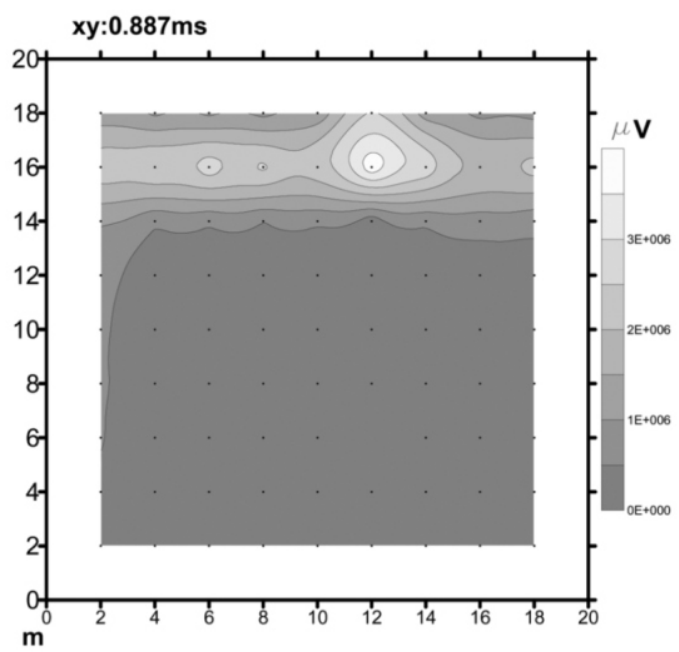

(c)

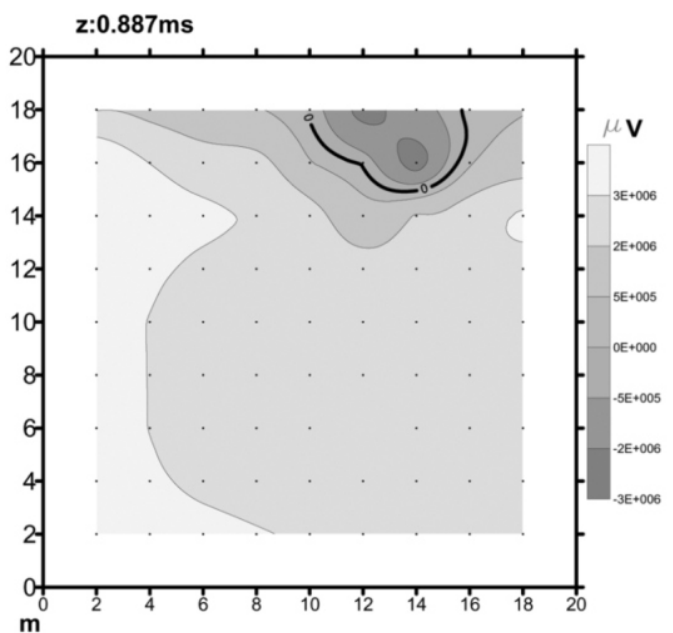

$x y: 2.087 \mathrm{~ms}$

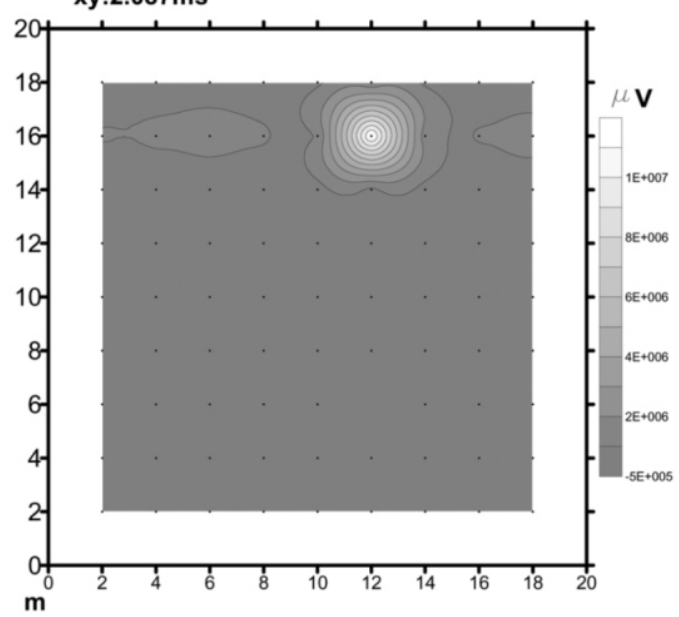

$\mathrm{z}: 2.087 \mathrm{~ms}$

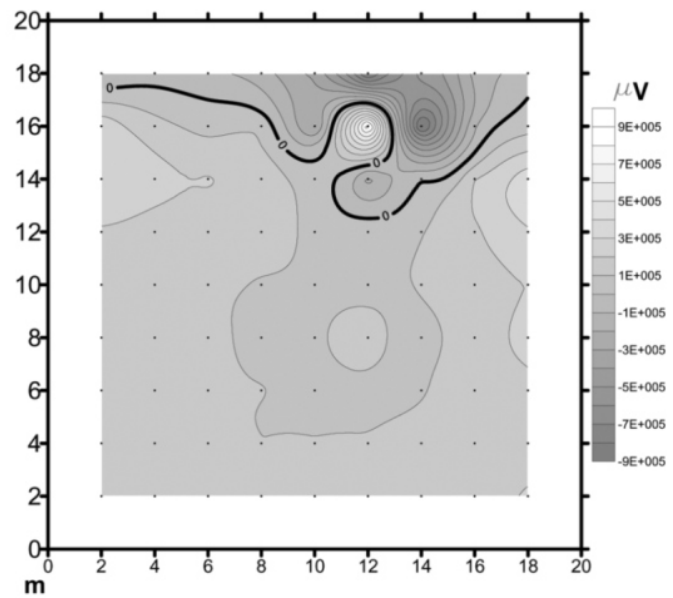

Fig. 6. Measured three-component transient voltages (in $\mu \mathrm{V}$ ) per amperes for the TEM map around a horizontal pipe. (a) A buried power line at depth of about $1 \mathrm{~m}$ that comes into the survey grid from the west, connects to the electrical access cover $(\mathrm{x}=12 \mathrm{~m}, \mathrm{y}=16 \mathrm{~m})$, and continues out of the grid to the east toward another light pole. (b) Horizontal component TEM survey results in planar view at times 0.887 and $2.087 \mathrm{~ms}$. An east-west trending anomalous zone at early time crossing through the respected grid and diminished at late time. (c) Vertical component TEM survey results in planar view for the same area at times 0.887 and $2.087 \mathrm{~ms}$ : weak response for pipes, while strong response for surface cover. 
plays only uni-polar anomalies, contain stronger anomaly data, and provide a good indication of a given target's location. However, there is a good deal of useful information in the horizontal components of near-surface TEM surveys, especially with regard to targeting horizontal orientation. Although Hx and Hy data are weaker for the horizontal transmitter loop, such data not only provide the best indication of target location, but also can be used to determine size, orientation, and characteristics of given targets.

Our experimental targets (well casings) were surrounded by other conductive materials. Consequently, images of the metal casings displayed are not as sharp as they would otherwise be. Inductive coupling for plate refers to the EM effect observed in a body when a secondary field is created by the current flowing in it. If the secondary field is derived from two, or more, energizing sources, such as conductive host contributions, the computation of such fields must account for geometric and time factors. The code we used can simulate these complex field interactions.

\subsection{Comparison between $B$ and $\mathrm{dB} / \mathrm{dt}$}

The traditional sensor used in TEM systems is an induction coil. This sensor measures voltage response proportional to the time rate of change of a magnetic field in the EM bandwidth. Using a SIROTEM TEM, all of our modeling and field experiments, $\mathrm{x}, \mathrm{y}$, and $\mathrm{z}$ components of the EMF (voltage, dB/dt) are considered. Smith and Annan (2000) compared these two forms of data ( $\mathrm{dB} / \mathrm{dt}$ and B-field) by examining power spectra model data and field examples, which all hade quite different characteristics. The power spectrum of the B-field excitation waveform had significantly more energy than the $\mathrm{dB} / \mathrm{dt}$ power spectrum at low frequencies, while at high frequencies the $\mathrm{dB} / \mathrm{dt}$ spectrum had more energy. The impact of this different distribution of energy can be seen in model data: the signal-to-noise ratio for good conductors is significantly enhanced in B-field data and reduced in $\mathrm{dB} / \mathrm{dt}$ data. Magnetic-field data is better for identifying, discriminating, and interpreting good conductors, while suppressing less conductive targets. Smith and Annan (2000) suggested an indirect measurement of a magnetic field in the same bandwidth by integrating the digitized output voltage from the induction coil. This method is becoming an interesting research trend in TEM.

\subsection{Comparison between Time Domain and Frequency Domain}

Nowadays, rapid improvements in measuring equipment, such as the 24-bit system for the frequency domain EM method make it possible to apply frequency domain EM measurement to shallow environments for mapping and detection work. It is worth noting that, theoretically, the complete transient waveform is mathematically equivalent to measuring the response at all frequencies for a harmonically varying source. Although the transient curve involves a wide frequency spectrum, the response measured at different times contains different proportions of high-frequency and low-frequency components. In general, at early times the response is due to both the low and high -frequency components; whereas at later times the low-frequency response is dominant. However, the behavior of harmonic and transient fields in low frequency and late regimes is fundamentally different. At the late stage of buildup, the transient field is more sensitive to changes in conductivity than components in the frequency domain. This is of fundamental importance in the resolving capabilities of the two methods (Kaufman 1978).

Undoubtedly, there exist some factors that may cause the TEM technique to fail: (1) High cultural background noise levels might overwhelm the anomaly. To increase the signal-to-noise ratio, a higher magnetic dipole moment for the transmitter is advise. This can be achieved by including higher transmitter currents, a larger transmitter loop, or using multiple-turn transmitter loops. (2) Missing an appropriate time windows can cause loss of a target signals; for example, our horizontal target (Fig. 6) detection experiment would have failed if we skipped the time window $\sim 0.887 \mathrm{~ms}$.

\section{CONCLUSION AND FUTURE WORK}

In this paper, we have demonstrated numerically that three-component TEM measurements can potentially give access to conductor information in conductive earth. We analyze 3-axis TEM data from a known well site and detect transient voltage anomalies, which are consistent with our theoretical modeling and which can be correlated with well locations in the conductor host. From this and other surveys, it is apparent that there is a lot of useful information in the horizontal components of near-surface TEM surveys. Whilst the vertical component contains stronger anomaly data, which displays only uni-polar anomalies, and provide the best indication on a given target's location, the horizontal component data, though weaker, can be used to determine size, orientation, and characteristics of targets, especially for targets extending horizontally (i.e., power lines, sewer pipes, etc.). Carlson and Zonge (2003) reached similar conclusions. We are encouraged by the correspondence between the TEM anomaly and the conductor information, and believe that the use of multi-component data to locate conductors will be an essential element of high-resolution EM methods used in engineering. Moreover, due to the benefits of better identifying, discriminating, and interpreting good conductors, indirect measurement of the magnetic field by integrating the digitized output voltage from the induction coil has become an urgent requirement of future TEM work. 
Acknowledgements The authors are grateful to the diligent field crew of the geoelectric group in the Department of Earth Sciences of National Central University (NCU). Many thanks are extended to professor Chia-Shyun Chen, the Institute of Apply Geology, NCU, for the support of the facility of the Groundwater Research Well Field on NCU campus. This study was partly supported by the grant NSC 96-2119-M-008-010. Valuable comments and suggestions made by anonymous reviewers are highly appreciated.

\section{REFERENCES}

Buselli, G. and B. O’Neill, 1977: SIROTEM - A new portable instrument for multichannel transient electromagnetic measurements. Explor. Geophys., Bull. Aust. Soc. Explor. Geophys., 8, 82-87, doi: 10.1071/EG977082. [Link]

Carlson, N. R. and K. L. Zonge, 2003: Minerals exploration methods modified for environmental targets. Explor. Geophys., Bull. Aust. Soc. Explor. Geophys., 34, 114-119, doi: 10.1071/EG03114. [Link]

Chen, C. S. and C. C. Chang, 2002: Use of cumulative volume of constant-head injection test to estimate aquifer parameters with skin effects: Field experiment and data an- alysis. Water Resour. Res., 38, 1056, doi: 10.1029/2001 WR000300. [Link]

Johansen, H. K. and K. Sorensen, 1979: Fast Hankel transforms. Geophys. Prospect., 27, 876-901, doi: 10.1111/j.13652478.1979.tb01005.x. [Link]

Kaufman, A., 1978: Frequency and transient response of electromagnetic fields created by currents in confined conductors. Geophysicsts, 43, 1002-1010, doi: 10.1190/1. 1440861. [Link]

Nabighian, M. N., 1991: Electromagnetic methods in applied geophysics: Application. Soc. Explor. Geophys., 2, 425 pp.

Lee, T., 1977: Estimate of depth to conductors by the use of electromagnetic transients. Geophys. Prospect., 25, 61-75, doi: 10.1111/j.1365-2478.1977.tb01153.x. [Link]

Raiche, A. and F. Sugeng, 1989: Predicting the transient EM response of complex structures using the compact finite element method. Explor. Geophys., Bull. Aust. Soc. Explor. Geophys., 20, 51-55, doi: 10.1071/EG989051. [Link]

Smith, R. S. and A. P. Annan, 2000: Using an induction coil sensor to indirectly measure the B-field response in the bandwidth of the transient electromagnetic method. Geophysics, 65, 1489-1494, doi: 10.1190/1.1444837. [Link] 